\title{
ANTIMICROBIAL ACTIVITY OF SUPPORTED METAL NANOPARTICLES:
}

\section{SILVER NANOPARTICLES}

\author{
S. R. Thakare, A. N. Ingole, and D. B. Patil \\ Department of Chemistry, Govt. Institute of Science, R.T. Road, \\ Civil Lines, Nagpur - 440001 (MS) India. Email \\ : sanjaythakare@yahoo.co.uk
}

\begin{abstract}
:
Ag nanoparticles are used in antimicrobial applications as Ag ions has antimicrobial activity synthesis of $\mathrm{Ag}$ nanoparticles in solution phase chemical reduction of $\mathrm{AgNO}_{3}$ with $\mathrm{NaBH}_{4}$ in presence of GO suspension. The size and shape of the Ag nanoparticles are dependent on the concentration of Ag salt. The nanoparticles of different shape such as spherical, triangular, pentagonal, ellipsoid and rod are obtained at the higher concentration of the Ag salt. The synthesized Ag nanoparticles show good antimicrobial activity against the Gram negative bacteria E. coli and P. aeruginosa. The synthesized Ag nanoparticles can be useful for several applications in biological area as well as materials science.
\end{abstract}

\section{Introduction:}

Metallic nanoparticles play an important role in wide number of applications in medical or biological applications. Ag nanoparticles are used in antimicrobial applications as Ag ions has antimicrobial activity [1-5]. Ag nanoparticles also showed cytoprotective activity towards HIV-1 infected cells [6]. It is being used in the medical field as well as in water and air filtration. Hybrid materials of Ag nanoparticles with amphiphilic hyper branched macromolecules are synthesized for use in surface coatings because of its antibacterial activity. The surfaces coated with Ag nanoparticles embedded paint based on vegetable oil showed excellent antimicrobial properties. The water related diseases like diarrhea and dehydration can be reduced by improving the microbial quality 
of the drinking water. The bactericidal activity can be reduced by the use of the Ag deposited carbon filters. The use of Ag coated activated carbon filter (ACF) effectively removes bioaerosols.

Graphene, a single layer of carbon atoms closely packed into honeycomb twodimensional (2D) lattice. Its $\mathrm{sp}^{2}$ carbon networks as well as extraordinary electronic, thermal and mechanical properties are interesting for wide range of applications [7-10]. Graphite oxide (GO) is a basic material for the preparation of graphene sheets in bulk quantity. Its large surface area, oxygen containing surface functionalities such as hydroxyl, carboxylic, carbonyl, epoxide groups and high water solubility are promising for many applications. Its inherent specific surface area as high as

$2600 \mathrm{~m}^{2} \mathrm{~g}^{-1}$ makes graphene particularly suitable to load catalyst nanoparticles. A variety of metal NPs such as Pd, Pt, Au, Ag and

alloys have been deposited on graphene nanosheets (GNs) or GO, exhibiting substantial performance improvements relative to the traditional catalysts. Despite these significant advances, it is recognized that the size and morphology of the NPs on GNs would be controlled carefully upon trying to optimize the catalytic performance $[11,12]$. This largely depends on the number and spatial distribution of oxygen functional groups that provide active sites for nucleation and growth of metal nanoparticls. For the highly oxidized GO the immobilization effect of defects is favorable to prevent small metal NPs from aggregation, while for the lightly oxidized GNs or chemically converted GNs, it is usually difficult to achieve uniform deposition of the metal NPs on the surface through a solution deposition process.

For instance, suspension based on functionalized sheets of graphene or graphene oxide (GO) provides a convenient route to keep sheets exfoliated and available for ion or nanoparticle 
intercalation. However, recent attention has been drawn towards the antimicrobial activity of graphene and its composites materials. Shen et al recently reported the Ag-chemically converted graphene (CCG) nanocomposite that shows very good antibacterial activity against Colibacillus, S. aureus and C. albicans [13]. Ag-CCG shows the high disinfection ability and completely destroys the bacterial cultures.

To control the dimension and morphology of the metal NPs on GNs, polymers have been employed to control the deposition and growth of the metal NPs on GNs, including proteins, DNA, Nafion, polyvinylpyrrolidone, polypyrrole and other synthetic polyelectrolytes (PEs). Compared to bare GO sheets, polymers adsorbed on the GN surface provide more active sites for immobilization of metal ions, and also afford an additional means to control the growth of NPs. In this regard, PEs are attractive due to their good dispersing capability on GNs and strong affinity to metal NPs [14, 15]. Exploiting these features, some studies have demonstrated that the loading amount of metal NPs on GNs was able to be adjusted through changing the mass ratio of $\mathrm{PE} / \mathrm{GNs}$ to the metal precursors, and their size and spatial distributions on $\mathrm{PE} / \mathrm{GNs}$ were also improved significantly. By comparison with the PEs lying flat on GNs, PE brushes where one end is covalently attached on the GN surface are expected to introduce more active sites for the immobilization of metal NPs. In particular, PE chains anchored on GNs could be more effective in confining the motion of NPs as opposed to those adsorbed PE chains and thus more homogeneous spatial distribution, when considering the possible influence of the added multivalent ions and solvent quality on the conformation of adsorbed PE chains . 
PE brushes are particularly suitable for the deposition of narrowly sizedistributed metal NPs due to the presence of a specific local environment, called a nnanoreactor', where the concentration of metal ions is far higher than that of the bulk solution. The relatively higher local concentration within brushes can induce rapid nucleation of metal NPs, and the subsequent growth and morphological evolution can be controlled by the diffusion of outside metal ions into the Nano reactor. Besides preventing NPs from aggregation, PE brushes also facilitate recycling and separation of NPs from the product. For instance, the metal NPs within spherical PE brushes can not only exhibit good dispersion stability but also keep the catalysis activity nearly unchanged even after multiple times of consecutive use. Compared to the spherical

PE brushes, GN-based brushes have larger specific surface areas and thus are expected to load more metal NPs. Furthermore, larger lateral sizes than spherical brushes enable them more easily to separate from the resulting products.

It is desire to exploit the unique biomedical properties of Ag nanoparticles led us to synthesis Ag nanoparticles on GO matrix.

We believe that the hybrid material of $\mathrm{Ag}$ nanoparticles where a layered material like GO is used as matrix can induce binding capability that usually lacks in Ag nanoparticles alone and thus enhance its antimicrobial activity. Zhou et al. reported the one step in situ synthesis of Ag nanoparticles on GO or reduced GO substrate without using any surfactant or reducing agent [16]. GO substrate was prepared by adsorption of GO on 3-aminopropyltriethoxysilane (APTES) modified SiOx substrate. This substrate was immersed in an aqueous solution of $\mathrm{AgNO}_{3}$ under $\mathrm{N}_{2}$ protection and heating at $75^{\circ} \mathrm{C}$. The electron in the negatively charge GO substrate is participated for the reduction of Ag ion to 
get the metallic Ag. Recently Shen et al. reported the solution based synthesis of Ag nanoparticles in CCG suspension with mixed reducing agent, ethylene glycol and $\mathrm{NaBH}_{4}$ [13]. They have investigated the SERS activity and the antibacterial activity of the synthesized Ag-CCG composite. Here we report solution phase synthesis of $\mathrm{Ag}$ nanoparticles by reduction of $\mathrm{AgNO}_{3}$ on $\mathrm{GO}$ matrix with $\mathrm{NaBH}_{4}$ as a reducing agent at normal atmospheric conditions. The antimicrobial activity of $\mathrm{Ag}$ nanoparticles on $\mathrm{GO}$ is also studied in details against the Gram negative bacteria Escherichia coli (E. coli) and Pseudomonous aeruginosa (P.aeruginosa).

Experimental:

\subsection{Materials}

Graphite powder (<20 micron) was purchased from fine chemicals and used . $\mathrm{AgNO}_{3}$ (99.8\%, fine chemicals, India), $\mathrm{NaBH}_{4}$ (>99\%,

S.D. Fine, India), hydrochloric acid (AR grade), $\mathrm{H}_{2} \mathrm{O}_{2}$, (merk chemicals), $\mathrm{KMnO}_{4}$ (>99\%, NICE-Chemical, India), nutrient broth (g/1, peptone $5 \mathrm{~g}$, beef extract $3 \mathrm{~g}$, sodium chloride $5 \mathrm{~g}, \mathrm{pH} 7$,

HiMedia, Mumbai, India) were used as-received and without any further purification.

2.2 Synthesis of Graphene oxide

$500 \mathrm{mg} \mathrm{NaNO}_{3}$ add slowly in the conc. $23 \mathrm{ml} \mathrm{H}_{2} \mathrm{SO}_{4}$ then one gm Graphite added slowly with stirring and cooling to keep the temperature of the reaction mixture below $10^{\circ} \mathrm{C}$. At this condition add $3 \mathrm{gm} \mathrm{KMnO}_{4}$ slowly, reddish grey colour was obtained. The temperature of the reaction mixture was increased and maintained at $35^{\circ} \mathrm{C}$ for $1 \mathrm{~h} .46 \mathrm{~mL}$ of deionised water was slowly added to this mixture then the temperature was increased upto $98^{\circ} \mathrm{C}$. After 15 minutes $140 \mathrm{~mL}$ of deionised water was again added followed by $10 \mathrm{~mL}$ of $30 \%$ $\mathrm{H}_{2} \mathrm{O}_{2}$ solution. The solid product was separated by 
centrifugation. It was washed repeatedly with $5 \% \mathrm{HCl}$ solution until the sulfate ions are removed and then washed with distilled water repeatedly until it becomes free of chloride ions. The product was then filtered and washed 3-4 times with acetone to make it moisture free and the residue thus obtained was dried in an air oven below at $65^{\circ} \mathrm{C}$ for overnight. The GO was suspended in water and exfoliated through ultrasonication for $3 \mathrm{~h}$. A homogeneous reddish brown solution of GO nanosheets was obtained. This GO-water suspension was used as a matrix for the preparation of the Ag nanoparticles.

\subsection{Preparation of Ag nanoparticles on GO}

$\mathrm{Ag}$ nanoparticles were prepared by reducing $\mathrm{AgNO}_{3}$ with $\mathrm{NaBH}_{4}$

in presence of GO suspension with starch colloidal solution. In

the typical procedure, $10 \mathrm{~mL}$ of homogeneous suspension of GO along with $1 \%$ starch solution was mixed with the desired amount of aqueous $\mathrm{AgNO}_{3}$ solution $\left(1 \times 10^{-3}\right.$ mol $\left.\mathrm{dm}^{-3}\right)$. Same procedure was adopted for other concentration of silver nitrate solution

The reaction mixture was stirred for $30 \mathrm{~min}$ at room temperature before addition of the reducing agent. $1 \mathrm{~mL}$ of $0.01 \mathrm{~mol} \mathrm{dm}^{-3}$ freshly prepared solution of $\mathrm{NaBH}_{4}$ was added slowly to the reaction mixture of $\mathrm{AgNO}_{3}-\mathrm{GO}$ suspension under vigorous stirring.

The colour of the reaction mixture turns into dark brown to grey depending on the concentration of the $\mathrm{AgNO}_{3}$. The reaction mixture was stirred for another 5 $\mathrm{h}$ for the complete reduction at room temperature.

\subsection{Characterization}

Preliminary characterization of formation of Ag nanoparticles was made by UVVisible spectroscopy. UV-Visible spectra were recorded in the range 200 to 800 nm using UV-3000 ${ }^{+}$. The Ag 
nanoparticle-GO suspension was diluted 10 times to record the UV-Visible spectra. The high resolution transmission electron microscopy (HRTEM) images were taken by a Philips CM200 model instrument operated at an accelerating voltage of $300 \mathrm{kV}$. Samples for HRTEM imaging were prepared by placing a drop of the solution sample in deionised water onto a carbon-coated $\mathrm{Cu}$ grid ( 3 $\mathrm{nm}$ thick, deposited on a commercial copper grid for electron microscope), dried in air and loaded into the electron microscopic chamber.

\section{Results and Discussion:}

The preliminary investigations of the

formation of the $\mathrm{Ag}$ nanoparticles on GO-water suspension are carried out by UV-Visible spectroscopy. The UV-Visible spectra of the $\mathrm{Ag}$ nanoparticles on GO-water suspension using different concentrations of aqueous $\mathrm{AgNO}_{3}$ solution are shown in Figure 1.

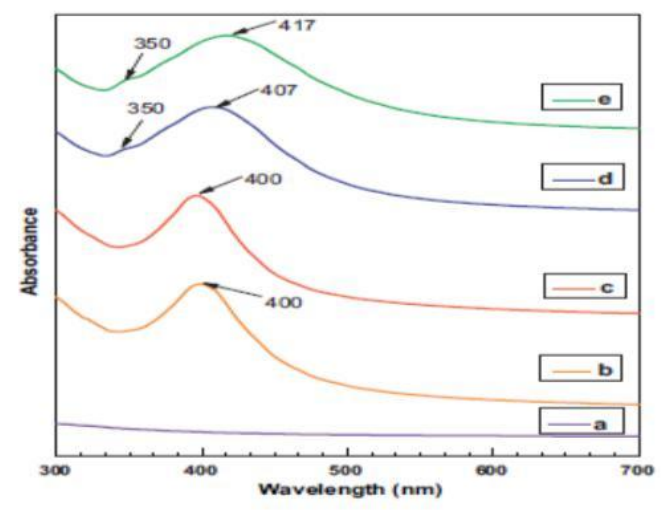

Figure1. The UV-Visible spectra of the Ag nanoparticles on GO-water

suspension using different concentrations of aqueous $\mathrm{AgNO}_{3}$. a-No $\mathrm{AgNO}_{3}, \mathrm{~b}-1, \mathrm{c}-2, \mathrm{~d}-3, \mathrm{e}-4\left(\times 10^{-3} \mathrm{~mol} \mathrm{dm}^{-3}\right.$ of $\left.\mathrm{AgNO}_{3}\right)$ 
The appearance of characteristic surface plasmon band at $400 \mathrm{~nm}$ indicates the formation of Ag nanoparticles on GO water suspension [17]. The surface plasmon band of the $\mathrm{Ag}$ nanoparticles is appeared at $400 \mathrm{~nm}$ when $1 \times 10^{-3}$ mol dm ${ }^{-3}$ of $\mathrm{AgNO}_{3}$ solutions are used to prepare $\mathrm{Ag}$ nanoparticles. However, the absorption band is shifted to longer wavelength in case of higher concentrations of $\mathrm{AgNO}_{3}$. The shifting of the absorption peak towards longer wavelength for the higher concentration of $\mathrm{AgNO}_{3}$ indicates the formation of larger Ag nanoparticles, with different shapes and sizes. This is also supported by transmission electron micro scope (TEM) analysis. Usually surface plasmon band shifts depending on the particle size, shape, chemical surrounding, adsorbed species on the surface and dielectric constant.
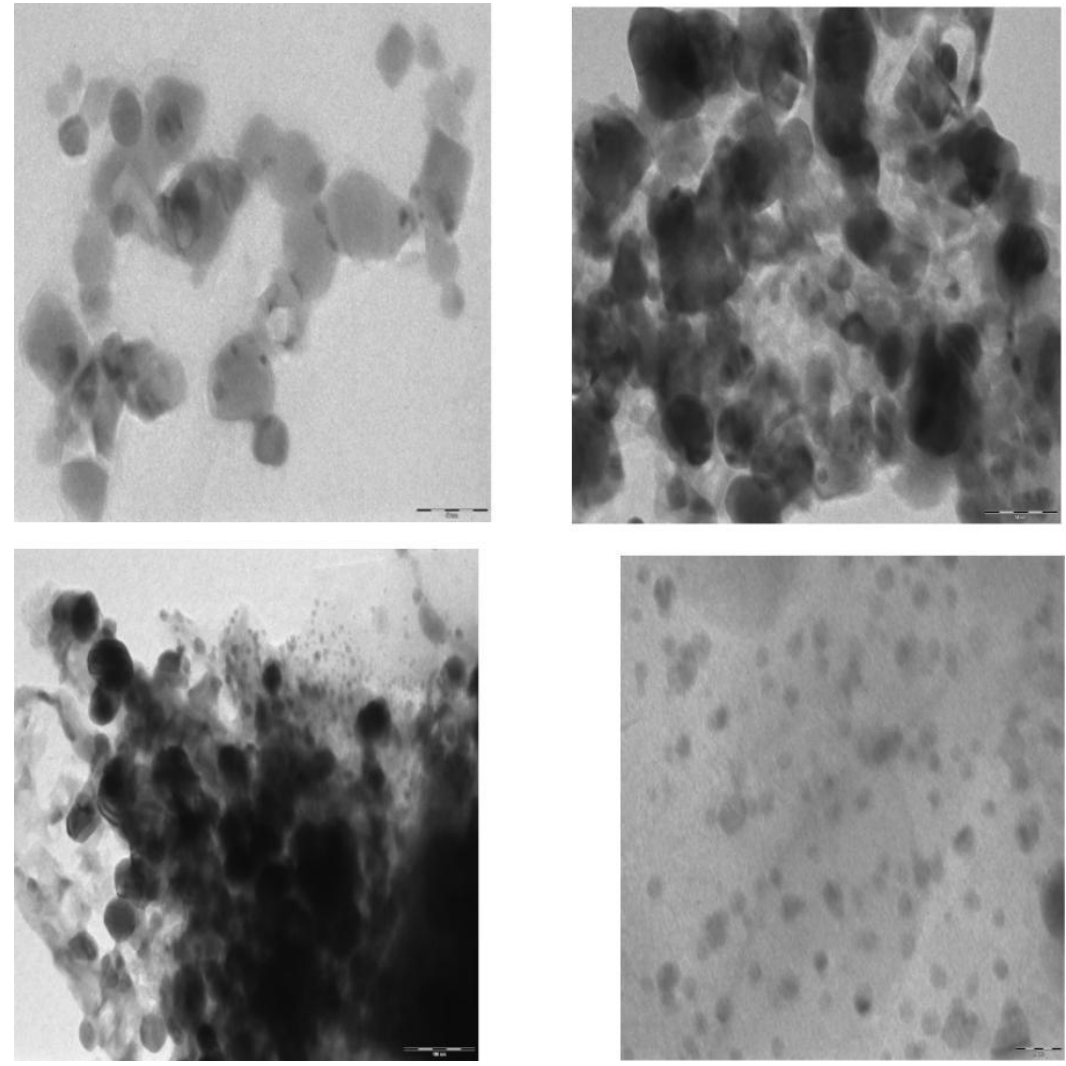

Figure 2. TEM images of Ag nanoparticles 
TEM images of Ag nanoparticles (Figure 2) show the wide distribution of particles ranging in the diameter of $5-25 \mathrm{~nm}$.

Figure 2 shows that the smaller particles are spherical in shape where as the larger nanoparticles are in an elongated form. The elongated shape could be the result of the aggregation of the two or more particles together. The size and shape of the Ag nanoparticles are also influenced by the concentration of $\mathrm{AgNO}_{3}$ solution. Most of the nanoparticles are spherical in shape when synthesized using 1

$\times 10^{-3} \mathrm{~mol} \mathrm{dm}^{-3}$ of $\mathrm{AgNO}_{3}$ solution. Synthesis of the $\mathrm{Ag}$ nanoparticles using higher concentration of $\mathrm{Ag}$ salt produces variable particle shapes. At this concentration nanoparticle with different shapes such as spherical, triangular, pentagonal, ellipsoid and rod are obtained. It indicates the crystalline nature of the Ag nanoparticles. The HRTEM images of Ag nanoparticles embedded on the GO sheets are shown in Figure 2 TEM image of the transparent silk waves of the GO sheets indicates that the Ag nanoparticles are deposited on the GO support.

There are few reports on the Ag nanoparticles decorated on the GO sheets. Recently Shen et al reported the solution based synthesis of Ag nanoparticles in CCG using mixed reducing agent and found the homogeneous distribution of Ag nanoparticles of size range 5 -

$10 \mathrm{~nm}$ on CCG [13]. The average crystallite size of the Ag nanoparticles is supported by the TEM analysis. Pasricha et al also reported the solution-based synthesis of Ag nanoparticles into

the GO sheets without using many reducing agent report it is confirmed by TEM analysis that the of size ranged 3-12 nm were decorated on to [15]. In this Ag nanoparticles the GO sheets. Formation of bigger particle due to agglomeration of the smaller particles was also observed. Lu et al. reported a novel synthesis method for the decoration of aerosol Ag nanocrystals on the GO sheet. The aerosol Ag nanocrystals were synthesized using a mini arc plasma reactor and subsequent deposition of as synthesized nanocrystals onto a GO. HRTEM analysis conformed that the Ag nanocrystals of about 5-10 nm were attached to the GO sheet. Zhou et al. also reported the single step in situ synthesis of Ag nanoparticles on single layer GO or reduced GO surfaces under thermal condition without any surfactant or reducing agent. This method resulted Ag nanoparticles in the size range of few nanometers to $1 \mathrm{~m}$ with non uniform size. Whereas the Ag 
nanoparticles, obtained by using solution phase synthesis reported in this study, are about 5-25 $\mathrm{nm}$ in size with variable shape. It is also observed that the shape of the nanoparticles is dependent on concentration of $\mathrm{AgNO}_{3}$ solution.

The bactericidal test was performed against two Gram negative bacteria, E. coli and $\mathrm{P}$. aeruginosa on nutrient agar plates containing $60 \mathrm{~L}$ of different Ag nanoparticle - GO hybrids. It was observed that both the bacterial strains were sensitive to synthesized Ag nanoparticles by using different concentration of $\mathrm{AgNO}_{3}$ and recorded zone of inhibitions from 7 to $26 \mathrm{~mm}$ (Fig.4).
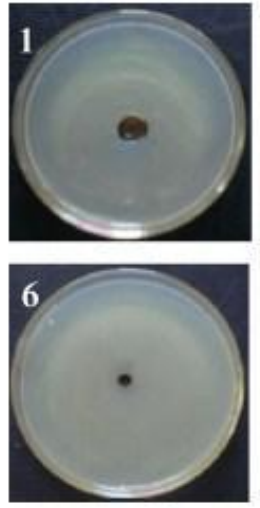
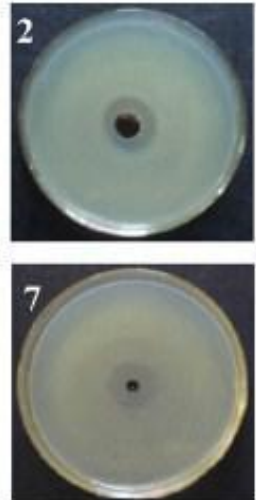
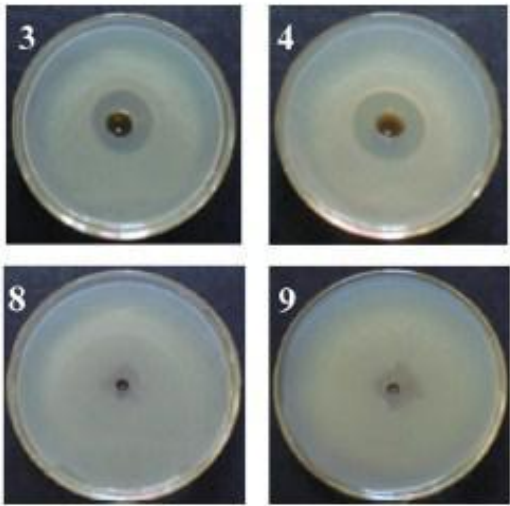
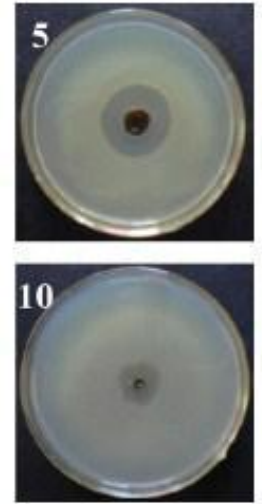

Figure 3. Bactericidal test. P. aeruginosa (1-5), E.Coli(6-10)

It was also observed that P. aeruginosa was comparatively more sensitive to the Ag nanoparticles and produced maximum growth inhibition zone $(26 \pm 1.53$ $\mathrm{mm}, \mathrm{n}=3, \mathrm{n}=$ numbers of experimental results). The detailed study pertaining to same is under progress.

\section{Conclusion :}

We have demonstrated the synthesis of Ag nanoparticles in solution phase chemical reduction of $\mathrm{AgNO}_{3}$ with $\mathrm{NaBH}_{4}$ in presence of $\mathrm{GO}$ suspension. The size and shape of the Ag nanoparticles are dependent on the concentration of Ag salt. The nanoparticles of different shape such as spherical, triangular, pentagonal, ellipsoid and rod are obtained at the higher concentration of the Ag salt. The synthesized Ag nanoparticles show good antimicrobial activity against the Gram negative bacteria E. coli and P. aeruginosa. The synthesized Ag 
nanoparticles can be useful for several applications in biological area as well as materials science.

\section{References:}

A. Frattini, N. Pellegri, D. Nicastro, O. de Sanctis, Mater. Chem. Phys. 94 (2005) 148.

P. Li, J. Li, C. Wu, Q. Wu, J. Li, J. Nanotechnol. 16 (2005) 1912.

I. Sondi, B. Salopek-Sondi, J. Colloid Interface Sci. 275 (2004) 177.

S. Pal, Y.K. Tak, J.M. Song, Appl. Environ. Microbiol. 73 (2007) 1712 .

V.K. Sharma, R.A. Yngard, Y. Lin, Adv. Colloid Interface Sci. 145 (2009) 83.

R.W.-Y. Sun, R. Chen, N.P.-Y. Chung, C.-M. Ho, C.-L.S. Lin, Chem.Commun. 40 (2005) 5059.

A.K. Geim, K.S. Novoselov, Nat. Mater. 6 (2007) 183.

K.S. Novoselov, A.K. Geim, S.V. Morozov, D. Jiang, M.I. Katsnelson, I.V. Grigorieva, S.V. Dubonos, A.A. Firsov, Nature 438 (2005) 197.

S. Stankovich, D.A. Dikin, G.H.B. Dommett, K.M. Kohlhaas, E.J.

Zinney, E.A. Stach, R.D. Piner, S.T. Nguyen, R.S. Ruoff, Nature 442 (2006) 282.

X. Li, X. Wang, L. Zhang, S. Lee, H. Dai, $\quad$ Science 319 (2008) 1229.

Z.S. Pillai, P.V. Kamat, J. Phys. Chem. B 108 (2004) 945.

S. Park, R.S. Ruoff, Nat. Nanotechnol. 4 (2009) 217.

J. Shen, M. Shi, N. Li, H. Ma, M. Ye, Nano Res. 3 (2010) 339. I.V. Ligtcap,

T.H. Kosel, P.V. Kamat, Nano Lett. 10 (2010) 577.

G.M. Scheuermann, L. Rumi, P. Steurer, W. Bannwarth, R. Mulhaupt, J. Am.Chem. Soc. 131 (2009) 8262.

X. Zhou, X. Huang, X. Qi, S. Wu, C. Xue, F.Y.C. Boey, Q. Yan, P.

Chen, H. Zhang, J.Phys. Chem. C 113 (2009) 10842.

F. Douglas, R. Yañ ez, J. Ros, J. Marin, A. De $\quad$ la. $\quad$ Escosura-

Mun iz, S. Alegret, $\quad$ A.Merkoc , J. Nanopart. Res. 10 (2008) 97. 